\title{
High-intensity exercise during chemotherapy induces beneficial effects 12 months into breast cancer survivorship
}

\author{
Sara Mijwel ${ }^{1,2}$ (D) $\cdot$ Anna Jervaeus $^{1} \cdot$ Kate A. Bolam $^{1} \cdot$ Jessica Norrbom $^{3} \cdot$ Jonas Bergh $^{2,4} \cdot$ Helene Rundqvist $^{5}$. \\ Yvonne Wengström ${ }^{1,2}$
}

Received: 12 October 2018 / Accepted: 27 February 2019 / Published online: 25 March 2019

(C) The Author(s) 2019

\begin{abstract}
Purpose Whether the benefits of exercise during chemotherapy continue into survivorship is not well-known. Here, the aim was to examine the effects of two exercise interventions on self-reported health-related and objectively measured physiological outcomes 12 months following commencement of chemotherapy.

Methods Two hundred and forty women with breast cancer stage I-IIIa were randomized to 16 weeks of high-intensity aerobic interval training combined with either resistance training (RT-HIIT), or moderate-intensity aerobic training (AT-HIIT), or to usual care (UC). Primary outcome: cancer-related fatigue (CRF); secondary outcomes: quality of life (QoL), symptom burden, muscle strength, cardiorespiratory-fitness, body mass, and return to work.

Results Compared to UC, both RT-HIIT and AT-HIIT significantly counteracted increases in total CRF (ES $=-0.34$; ES $=-$ 0.10 ), daily life $\mathrm{CRF}$ ( $\mathrm{ES}=-0.76$; $\mathrm{ES}=-0.50$, and affective $\mathrm{CRF}$ ( $\mathrm{ES}=-0.60$; $\mathrm{ES}=-0.39$ ). Both RT-HIIT and AT-HIT reported significantly lower total symptoms $(\mathrm{ES}=-0.46, \mathrm{ES}=-0.46)$, and displayed gains in lower limb $(\mathrm{ES}=0.73 ; \mathrm{ES}=1.03)$ and handgrip muscle strength (surgery side $\mathrm{ES}=0.70, \mathrm{ES}=0.71$; non-surgery side $\mathrm{ES}=0.57, \mathrm{ES}=0.59$ ). AT-HIIT displayed significant reductions in body mass $(\mathrm{ES}=-0.24)$, improved $\mathrm{QoL}$ : role $(\mathrm{ES}=0.33)$ and emotional functioning $(\mathrm{ES}=0.40)$, and a larger proportion had returned to work $(p=0.02)$ vs UC.

Conclusion These findings emphasize the beneficial effects of supervised high-intensity exercise during chemotherapy to improve the health and to reduce societal costs associated with prolonged sick leave for patients with breast cancer several months following chemotherapy.

Implications for Cancer Survivors These findings provide important information with substantial positive consequences for breast cancer survivorship. High-intensity exercise programs during chemotherapy and support to maintain physical activity can be a powerful strategy to manage or prevent many of the short- and long-term adverse effects of treatment for the increasing cohort of cancer survivors.
\end{abstract}

Keywords Randomized controlled trial · Cancer-related fatigue $\cdot$ Breast cancer · High-intensity interval training · Cancer survivorship

Sara Mijwel

Sara.Mijwel@ki.se

1 Department of Neurobiology, Care Sciences and Society, Karolinska Institutet, Stockholm, Sweden

2 Cancer Theme, Karolinska University Hospital, Stockholm, Sweden

3 Department of Physiology and Pharmacology, Karolinska Institutet, Stockholm, Sweden

4 Department of Oncology and Pathology Cancer Center Karolinska, Karolinska Institutet, Stockholm, Sweden

5 Department of Cell and Molecular Biology, Karolinska Institutet, Stockholm, Sweden

\section{Introduction}

Advanced treatment strategies, which lead to improved survival for patients diagnosed with early stage breast cancer [1], also mean that more patients are at risk of suffering from toxic effects of chemotherapy regimens such as persistent debilitating symptoms that remain into survivorship [2]. During the survivorship period, which we here refer to as the period following primary breast cancer treatment in accordance with the physical activity and cancer control framework [3], cancerrelated fatigue (CRF) has been reported to be one of the most debilitating symptoms and can persist for years [4]. Moreover, CRF has been shown to be a major barrier to perform physical 
activity (PA), and affects physical and psychosocial wellbeing and ability to work [5]. Women who have undergone chemotherapy for breast cancer experience declines in cardiorespiratory fitness and muscular strength [6-8]. The results from the original 16-week OptiTrain study $[9,10]$ showed beneficial effects of high-intensity interval training, particularly in combination with resistance exercise, on cancer-related fatigue, symptoms, muscle strength, cardiorespiratory fitness, and body mass. Our findings are in line with a number of other trials [11]; however, few trials have reported on long-term effects of exercise during chemotherapy [12-17]. Here, the long-term effects of two different supervised exercise programs during chemotherapy were investigated with focus on objectively measured physiological and self-reported healthrelated outcomes. During the follow-up period, an effort was also made to support the participants to maintain physical exercise. At 12 months, compared to baseline, we hypothesized that both exercise groups would display sustained levels for health-related and physiological outcomes whereas the control group would show declined levels. The reason why sustained effects were expected were hypothesized to be due to an increased knowledge and awareness of the participants' physical capabilities that were gained from the supervised exercise sessions and due to the support to maintain physical activity during the follow-up period. The aim of this study was to investigate and compare the effects of the OptiTrain exercise interventions on the primary outcome: cancer-related fatigue and secondary outcomes: health-related quality of life (HRQoL), symptoms, physiological outcomes, and return to work 12 months following the commencement of chemotherapy in women with breast cancer, i.e. 9 months after completion of the intervention.

\section{Methods}

\section{Participants and procedures}

The methods of the OptiTrain randomized controlled trial (NCT02522260, Optimal Training Women with Breast Cancer (OptiTrain), www.clinicaltrials.gov) have been reported elsewhere [9, 10]. In brief, participants were recruited from two different oncology clinics in Stockholm, Sweden, from March 2013 to July 2016. Eligibility criteria included women (i) aged 18-70 years, (ii) diagnosed with I-IIIa stage breast cancer, and (iii) planned to receive adjuvant chemotherapy. Participants were randomized to 16 weeks of resistance training combined with high-intensity interval training (RT-HIIT), moderate-intensity aerobic training combined with highintensity interval training (AT-HIIT), or usual care (UC). The intervention groups (RT-HIIT and AT-HIIT) commenced the exercise training 3 days after the second chemotherapy session and ended the intervention 3 weeks following the last chemotherapy session. Details regarding the randomization process and blinding have been explained elsewhere [10]. Ethical approval was obtained from the Regional Ethical Review Board in Stockholm Sweden (Dnr 2012/1347-31/1, 2012/1347-31/2, 2013/7632-32, 2014/408-32) and was conducted in accordance with the Helsinki Declaration. All participants gave written informed consent.

The participant flow during and following the exercise trial is shown in Fig. 1. Participants were contacted by phone or e-mail for follow-up measurements, which took place 12 months following baseline assessments. The primary outcome was cancer-related fatigue and secondary outcomes included muscle strength, cardiorespiratory fitness, body mass, HRQoL, symptoms, and return to work. If participants agreed to participate, reassessments took place in the in-clinic gym where muscular strength (isometric mid-thigh pull and handgrip), estimated cardiorespiratory fitness (Åstrand-Rhyming submaximal cycle test), and body mass were measured as previously described [10]. Questionnaires were sent by post or electronically to those with e-mail addresses and consisted of the revised Piper Fatigue Scale (PFS) [18, 19], European Organization for Research and Cancer Treatment Quality of Life Questionnaire (EORTC-QLQ-C30) [20], and Memorial Symptom Assessment Scale (MSAS) [21]. A question regarding how much sick leave the participants were taking at 12 months was included with five possible options: $0 \%, 25 \%, 50 \%, 75 \%$, or $100 \%$, as well as a selfreported single item question of the participants' PA levels allowing categorization of participants as not meeting the exercise recommendations of at least $150 \mathrm{~min}$ of moderate-intensity exercise per week $[22,23]$ or meeting exercise recommendations at baseline, 16 weeks, and at 12 months. Objectively measured activity patterns were assessed only at baseline by an accelerometer (GT3X ActiGraph ${ }^{\circledR}$ Corp, Pensacola, FL, USA). Further details regarding methods and analysis have been explained elsewhere [9]. For the follow-up, procedures of the mentioned outcomes were identical to the original study $[9,10]$.

\section{Exercise training interventions}

The OptiTrain exercise protocol has been described previously $[9,24]$. The exercise groups undertook the supervised exercise sessions in an exercise clinic twice weekly for 16 weeks and the session duration was approximately $60 \mathrm{~min}$. HIIT was combined either with resistance training or incorporated as part of an overall endurance training session (AT-HIIT) in order to obtain similar exercise durations in both groups. In brief, the RT-HIIT group performed high-load resistance exercises targeting the major muscle groups consisting of two to three sets of 8-12 repetitions at an initial intensity of $70 \%$ of their estimated 


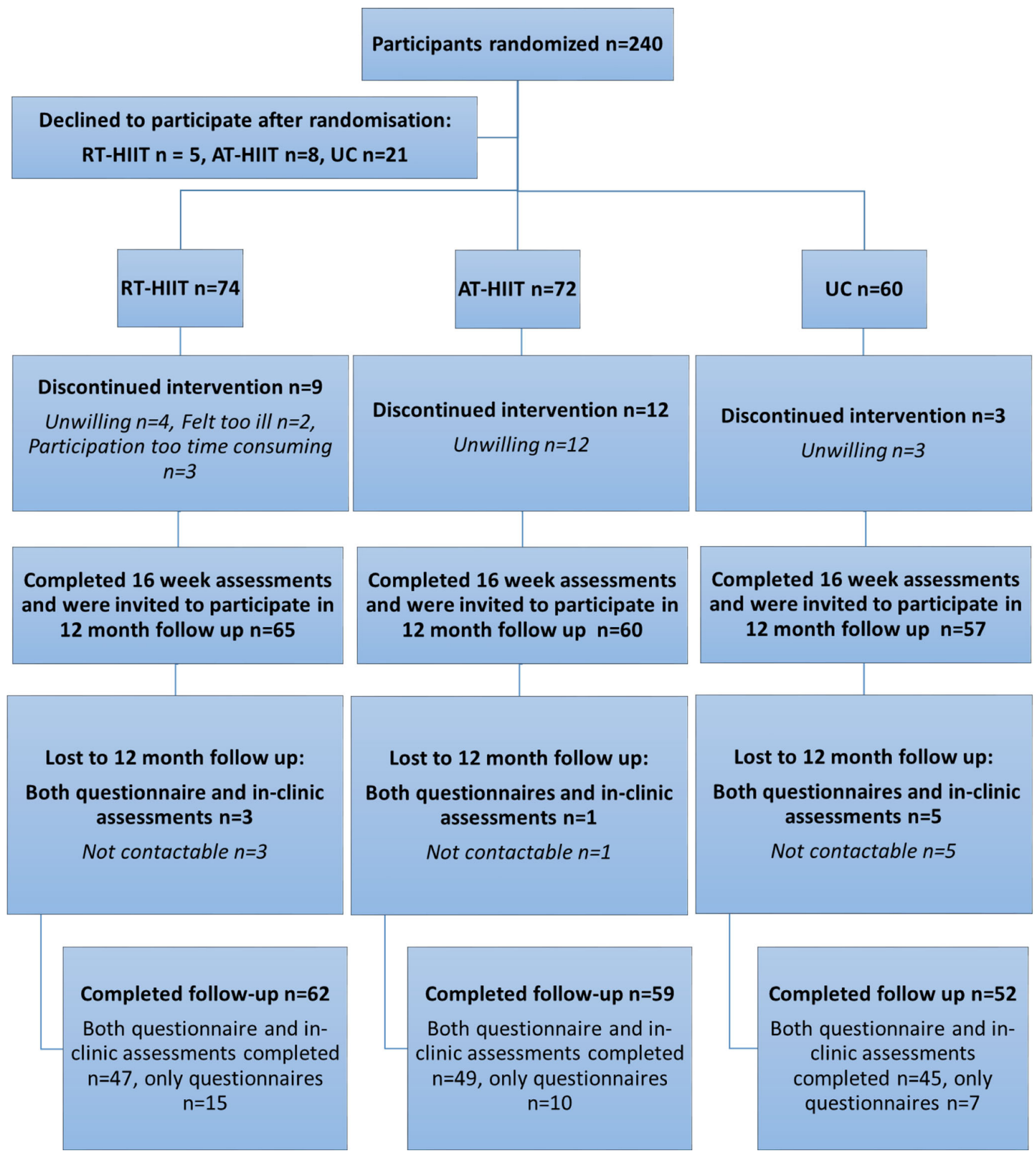

Fig. 1 Participant flow through the 12 month follow-up study

one repetition maximum (1-RM), progressing to $80 \%$ of 1-RM. The RT-HIIT sessions concluded with $3 \times 3$-min bouts of high-intensity interval exercise on a cycle ergometer interspersed with $1 \mathrm{~min}$ of recovery. The AT-HIIT group initiated each session with 20 min of moderateintensity continuous aerobic exercise followed by the same high-intensity interval exercise as in RT-HIIT. The UC group was given written information about physical activity at the initiation of the intervention period about exercise recommendations for patients with cancer according to the American College of Sports Medicine guidelines [23]. 


\section{Motivational seminars and exercise sessions to maintain physical exercise adherence}

Directly after the completion of the 16-week exercise program, participants in the exercise groups were offered a written, basic, PA on prescription by nurses involved in the exercise trial. The prescription was based on the text book Physical Activity in the Prevention and Treatment of Disease for evidence-based prescriptions [25]. During the follow-up, in collaboration with a national gym organization Friskis \& Svettis, these participants were offered a one-on-one exercise counseling session with a professional health educator which they were able to use at any time during the follow-up period and were offered the opportunity to purchase gym cards at a reduced rate. Participants were also invited to an additional two to three motivational exercise sessions per year during the follow-up period (seven in total during the follow-up period between 2014 and 2017) that included motivational presentations and physical exercise demonstrations organized by the research team in collaboration with Friskis \& Svettis. The UC group were not given any advice other than printed written information about exercise recommendations for patients with cancer according to the American College of Sports Medicine guidelines at the initiation of the intervention [22].

\section{Statistical analysis}

The calculation of the sample size was based on the primary outcome CRF and has previously been reported [9]. Variables were visually checked for normality through QQ-plots and skewness. Non-normally distributed data were log-transformed. Baseline and 12-month medical and demographic characteristics were summarized using descriptive statistics (Table 1). Exact $\chi^{2}$ tests were used for categorical variables and a one-way ANOVA for continuous data. Linear mixed model using unstructured covariance model was used to assess differences between groups at the 12-month follow-up, adjusted for baseline values of the outcome. For the primary outcome, the model was also adjusted for tumor receptor and menopausal status. Group-by-time interaction was included as a fixed effect. Since the model includes all available data (i.e., baseline, 16-weeks, and 12 months), intention to treat analysis was possible without imputing data. Data available from 16 weeks were included in the model but only presented as mean \pm SD in Tables 2, 3, 4, and 5. Adjustment for multiple groupwise comparisons were performed by using Bonferroni post hoc corrections. Effect sizes were calculated as the mean pre-post change in the treatment group minus the mean prepost change in the control group, divided by the pooled pretest standard deviation [26]. Pooling only the pretest standard deviation has been shown to provide an unbiased estimate of the effect size [26]. According to Cohen's guidelines, effect sizes with scores of $0.2-0.5,0.5-0.8$, and $>0.8$ were considered small, medium, and large effects, respectively [27]. Pearson's coefficient of correlation $(r)$ was used to evaluate the association between changes in physiological outcomes and changes in CRF. The statistical software used was SPSS version 24 (IBM corporation, Chicago, IL, USA). All tests were two-tailed and significance level was set at $p<0.05$.

\section{Results}

The time period for baseline and 12-month measurements ranged from March 2013 to July 2017. Attendance to the 16-week exercise intervention for participants in the RTHIIT and AT-HIIT groups were 68 and 63\%, respectively. At 12 months, $95 \%$ of the women who completed pre- and postmeasurements filled out all questionnaires and $78 \%$ agreed to come back for in-clinic physiological reassessments (Fig. 1). There were no differences between intervention groups regarding participant characteristics (Table 1). Those that completed in-clinic assessments (muscle strength, cardiorespiratory fitness, and body mass) had significantly more moderatevigorous PA min/day (objectively measured) at baseline $(p=0.03)$ and had significantly higher attendance rates during the intervention period compared to those that were lost to follow-up or completed the survey only $(p=0.005)$. However, there were no significant differences between the exercise groups and the UC group. Self-reported PA at baseline, 16 weeks and at 12 months are shown in Fig. 2. On average, $20 \%$ of the participants attended the motivational seminars $($ range $=11 \%-27 \%)$. Significant group $\times$ time (baseline to 12 months post-baseline) results are reported below.

\section{Primary outcome: cancer-related fatigue}

Changes in CRF assessed by the Piper Fatigue Scale are shown in Table 2 and in Fig. 3. At 12 months, both RT-HIIT and AT-HIIT were superior to UC for total CRF ( $\mathrm{ES}=-0.34$; $\mathrm{ES}=-0.10)$, affective/emotional $\mathrm{CRF}(\mathrm{ES}=-0.60 ; \mathrm{ES}=-$ 0.39 ), and behavior/daily life $\mathrm{CRF}(\mathrm{ES}=-0.76$; $\mathrm{ES}=-0.50$ ). AT-HIIT counteracted increases in cognitive CRF (ES $=-$ $0.13)$.

\section{Health-related quality of life}

Changes in HRQoL from the EORTC-QLQ-C30 are shown in Table 3. For the functional subscales on the EORTC-QLQ$\mathrm{C} 30$, role and emotional functioning favored AT-HIIT compared to $\mathrm{UC}(\mathrm{ES}=0.33$; $\mathrm{ES}=0.40)$. For the symptom subscale, AT-HIIT displayed favorable effects for fatigue (ES = $-0.40)$ and appetite loss $(\mathrm{ES}=-0.66)$ compared to UC. ATHIIT also had less constipation symptoms vs both RT-HIIT and $\mathrm{UC}(\mathrm{ES}=-0.28 ; \mathrm{ES}=-0.37)$. 
Table 1 Participant characteristics at baseline

\begin{tabular}{lllll}
\hline & $\begin{array}{l}\text { RT-HIT } n=74 \\
\text { Mean } \pm \text { SD }\end{array}$ & $\begin{array}{l}\text { AT-HIT } n=72 \\
\text { Mean } \pm \text { SD }\end{array}$ & $\begin{array}{l}\text { UC } n=60 \\
\text { Mean } \pm \text { SD }\end{array}$ & $p$ value* \\
\hline Age (years) & $52.7 \pm 10.3$ & $54.4 \pm 10.3$ & $52.6 \pm 10.2$ & 0.89 \\
Body mass (kg) & $68.7 \pm 11.3$ & $67.7 \pm 13.0$ & $69.1 \pm 11.0$ & 0.37 \\
Height (cm) & $165.7 \pm 6.7$ & $165.3 \pm 6.6$ & $166.4 \pm 7.0$ & 0.76 \\
SED (\% of daily wear time) & $63.7 \pm 7.7$ & $65.6 \pm 6.2$ & $66.6 \pm 7.2$ & 0.28 \\
MVPA (\% of daily wear time) & $9.6 \pm 2.8$ & $8.3 \pm 2.8$ & $8.5 \pm 4.3$ & 0.14 \\
& $\%$ & $\%$ & $\%$ & $p$ value† \\
Married or partnered & 60.6 & 59.7 & 69.5 & 0.81 \\
University completed & 67.6 & 64.7 & 66.0 & 1.00 \\
Postmenopausal women & 51.4 & 63.9 & 61.7 & 0.39 \\
Tumor receptor status & & & & 0.15 \\
Triple negative & 14.9 & 11.0 & 16.7 & 20.0 \\
HER2+, ER \pm & 21.6 & 30.2 & 61.6 & \\
HER2-, ER+ & 62.2 & 58.9 & 1.7 & 0.40 \\
HER2-, ER- & 1.4 & 0.0 & 70.5 & 0.98 \\
Hormone therapy & 85.7 & 84.4 & 58.3 & \\
Chemotherapy received at baseline & & 37.0 & & \\
Taxane-based therapy & 40.6 & 63.0 & & \\
Anthracycline-based therapy & 59.4 & & & \\
\hline
\end{tabular}

SD standard deviation, RT-HIIT resistance and high-intensity interval training, AT-HIIT moderate-intensity aerobic and high-intensity interval training, $U C$ usual care

*One-way ANOVA

${ }^{\dagger}$ Exact $\chi^{2}$ test

\section{Symptom burden}

Changes for symptoms as measured by the MSAS are shown in Table 4. At 12 months, both RT-HIIT and AT-HIIT reported significantly less total symptoms $(\mathrm{ES}=-0.46 ; \mathrm{ES}=-0.46)$ and physical symptoms $(\mathrm{ES}=-0.65 ; \mathrm{ES}=-0.61)$ compared to UC. Additionally, AT-HIIT reported lower symptom burden vs $\mathrm{UC}(\mathrm{ES}=-0.46)$.

\section{Muscle strength, cardiorespiratory fitness, and body mass}

Changes in physiological outcomes are shown in Table 5. At 12 months, significant lower-limb strength gains were found for both exercise groups compared to UC (RT-HIIT, ES = 0.73; AT-HIIT, ES = 1.03). Significant handgrip strength gains were also shown for both RT-HIIT (surgery side, ES $=0.70$; non-surgery side, ES $=0.57$ ) and AT-HIIT (surgery side, ES = 0.71; non-surgery side, $\mathrm{ES}=0.59$ ) vs UC. AT-HIIT displayed significant reductions in body mass compared to UC $(\mathrm{ES}=-$ $0.24)$.

\section{Sick leave/return to work}

At 12 months, the proportion of participants on more than half-time sick leave was significantly lower in the AT-HIIT group (5.9\%) compared to the UC group (31.0\%) ( $p=0.006)$. In the RT-HIIT group $17.0 \%$ were on more than half-time sick leave. The difference remained significant between AT-HIIT $(91 \%)$ and $\mathrm{UC}(69 \%)(p=0.020)$ when assessing the proportion of participants that had partially or completely returned to work (i.e., from $100 \%$ sick leave to $50 \%, 25 \%$, or $0 \%$ sick leave) compared to those that had not returned to work. In the RT-HIIT group, $82 \%$ had partially or completely returned to work.

\section{Associations between changes in CRF and changes in physiological outcomes}

Significant negative associations on changes were found between lower limb muscle strength and CRF assessed by the EORTC-QLQ-C30 $(r=-0.25, p=0.008)$, as well as CRF assessed by PFS $(r=-0.20, p=0.035)$. Moreover, cardiorespiratory fitness was negatively associated with CRF measured by PFS $(r=-0.25, p=0.005)$.

\section{Discussion}

This is the first study to assess if resistance or aerobic training combined with high-intensity interval training (HIIT) during chemotherapy can induce benefits that are present several 
months after a breast cancer diagnosis. 1 year following the commencement of the OptiTrain exercise trial, we found beneficial effects of both RT-HIIT as well as AT-HIIT on fatigue, muscle strength, and symptoms. AT-HIIT also resulted in beneficial effects on body mass, symptom burden, role and emotional functioning, as well as lower sick leave rates compared to UC. These findings provide novel and important information with substantial positive consequences for breast cancer survivorship and clinical implications.

At 12 months, both RT-HIIT and AT-HIIT were beneficial to counteract CRF with moderate/large effect sizes found for RT-HIIT regarding behavioral/daily life CRF and emotional/ affective CRF. Our findings are in contrast to previous followup trials consisting of combined resistance and aerobic training, which did not demonstrate sustained effects on fatigue at the 4-6 [12-15] or 12 month follow-up [28]. The discrepant results may be explained by the difference in information about PA given to the usual care group immediately following the interventions. In the current trial, the UC group is strictly treated as a control until the 5-year follow-up is completed. Moreover, the OptiTrain intervention is the first trial to provide physical activity prescriptions and motivational support to the exercise groups following the intervention, which may have resulted in inducing a behavioral change in the exercise groups as indicated by the self-reported PA data. Challenges to display beneficial effects of exercise training on cognitive and affective CRF has been evident in patients with breast cancer immediately following completion of chemotherapy [29]. Conversely, a trial conducted post-chemotherapy on breast cancer survivors assessing different exercise loads/intensities demonstrated that only the group performing high load resistance training combined with high-intensity continuous/ interval training was able to counteract mental fatigue [30], and results from their 12-month follow-up study showed that the higher intensity group was superior to lower intensity exercise on role functioning [31]. It may be speculated that the HIIT component in the current trial may have played a role in inducing beneficial effects for cognitive CRF found for ATHIIT, possibly through beneficial effects of HIIT on cerebral oxygenation [32] and/or improvements in information processing speed [33] as found in obese and elderly individuals, respectively. Despite low attendance at the motivational seminars, psychosocial factors that may have influenced outcomes for the exercise groups must be taken into account given that the UC group did not receive comparable motivational support. However, it must be noted that this finding may not be clinically meaningful due to the low effect size $(E S=0.13)$.

It remains unknown whether fatigue displayed during survivorship is due to cancer treatment, inactivity, or underlying symptoms/co-morbidities. Over the 16-week intervention, we found a significant negative association between changes in CRF and in lower-limb muscle strength [10]. The same association was found at 12 months, suggesting muscle weakness 


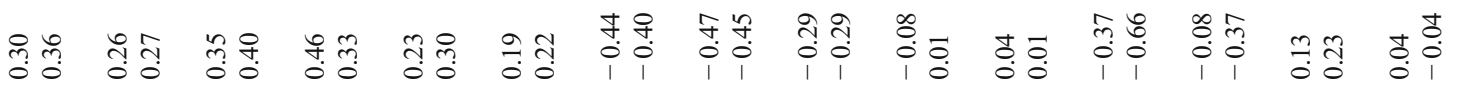
芯䒚

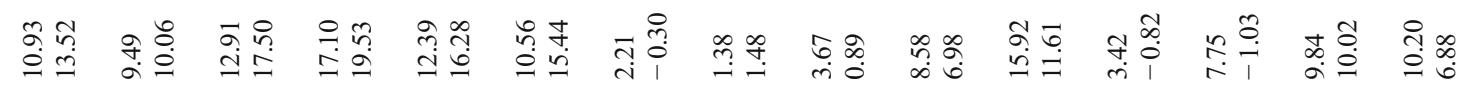

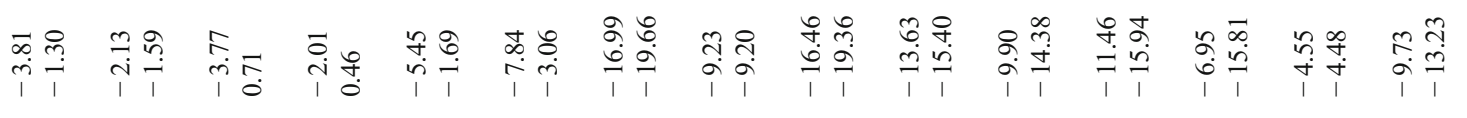

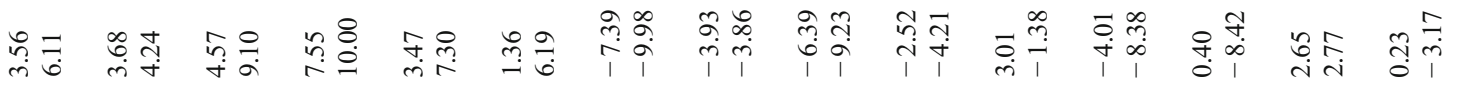

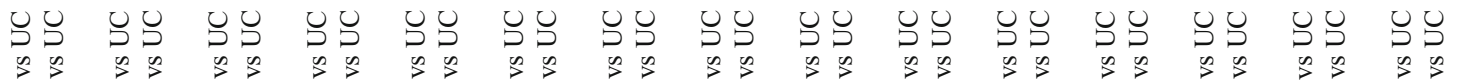

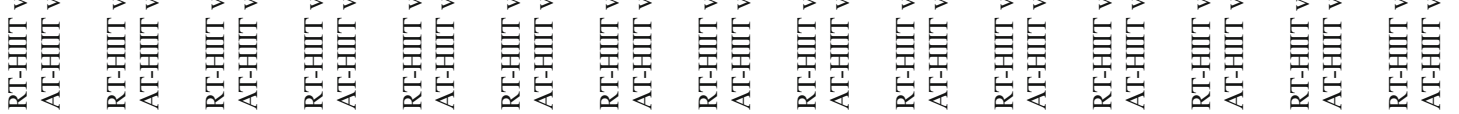

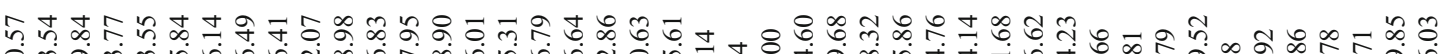

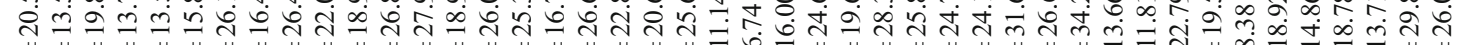
H $\begin{aligned} & H \text { H } \\ & \text { N }\end{aligned}$

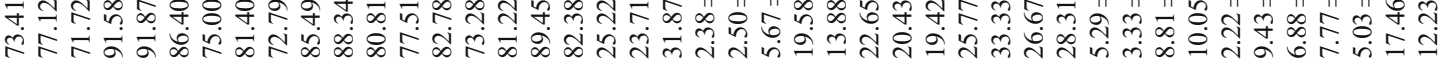
$\infty$ กิ

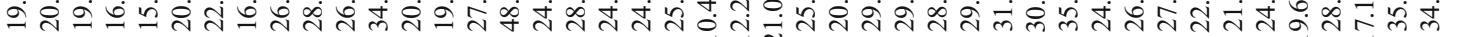

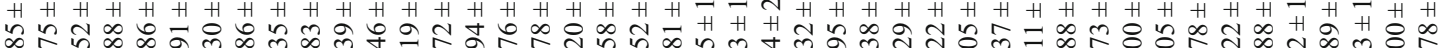

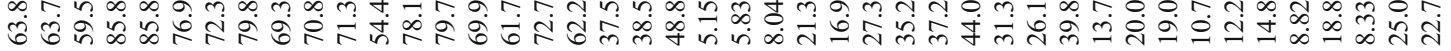

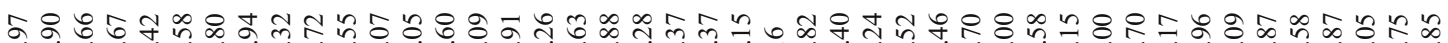

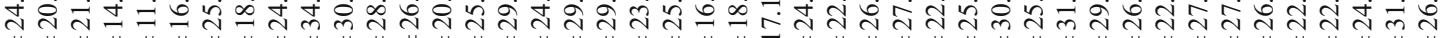

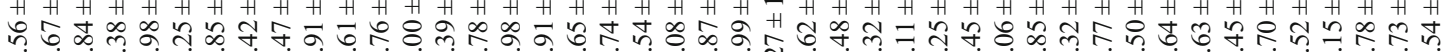
ठु

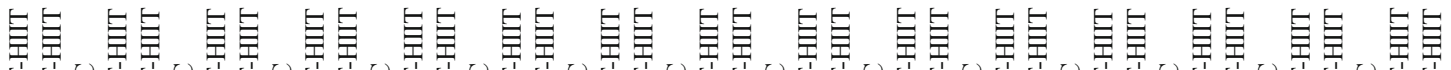

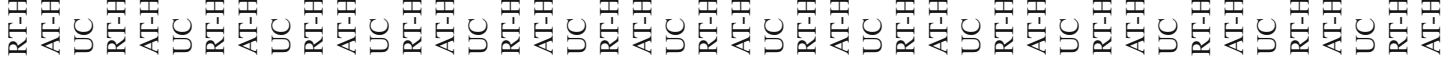




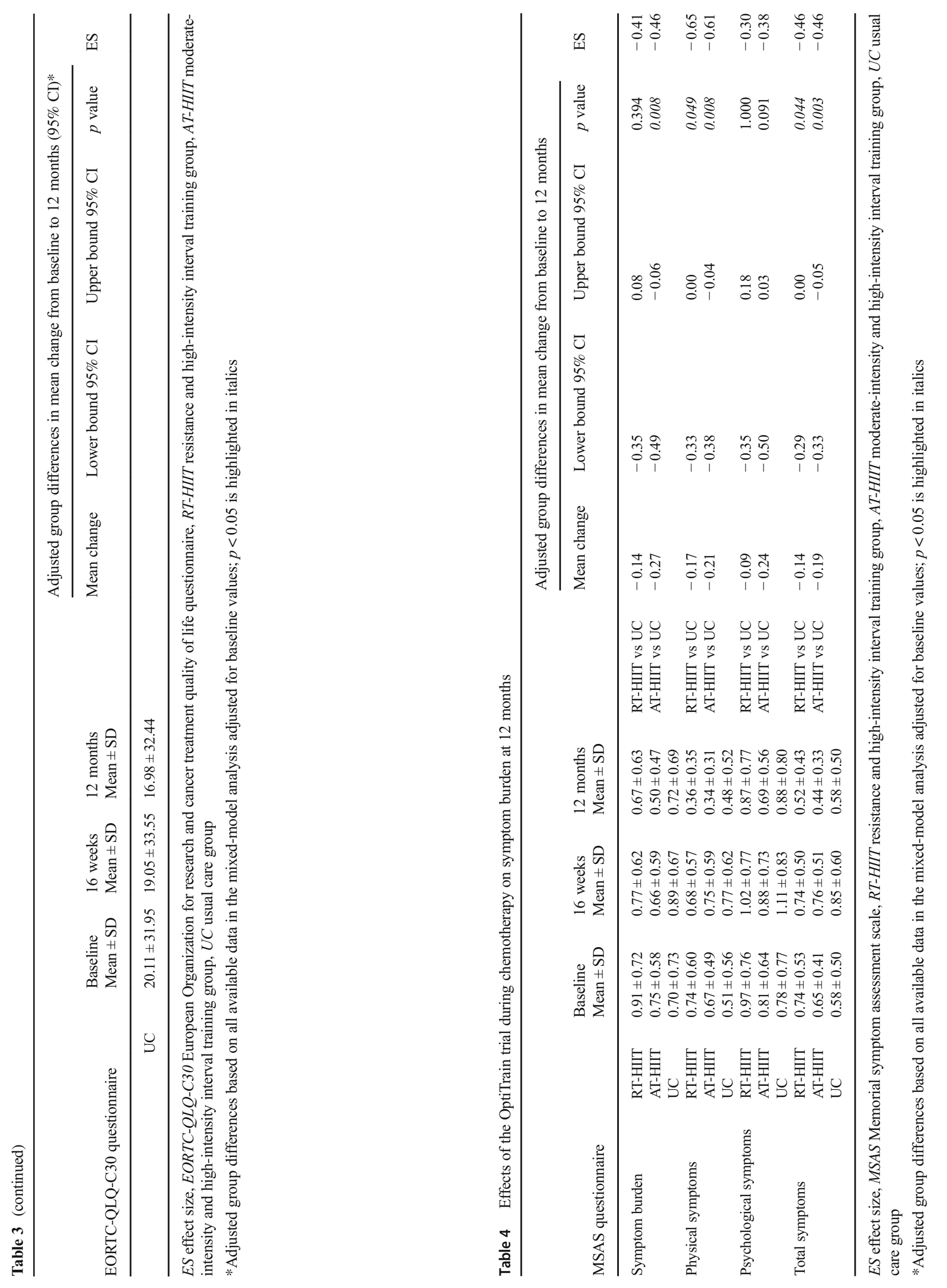




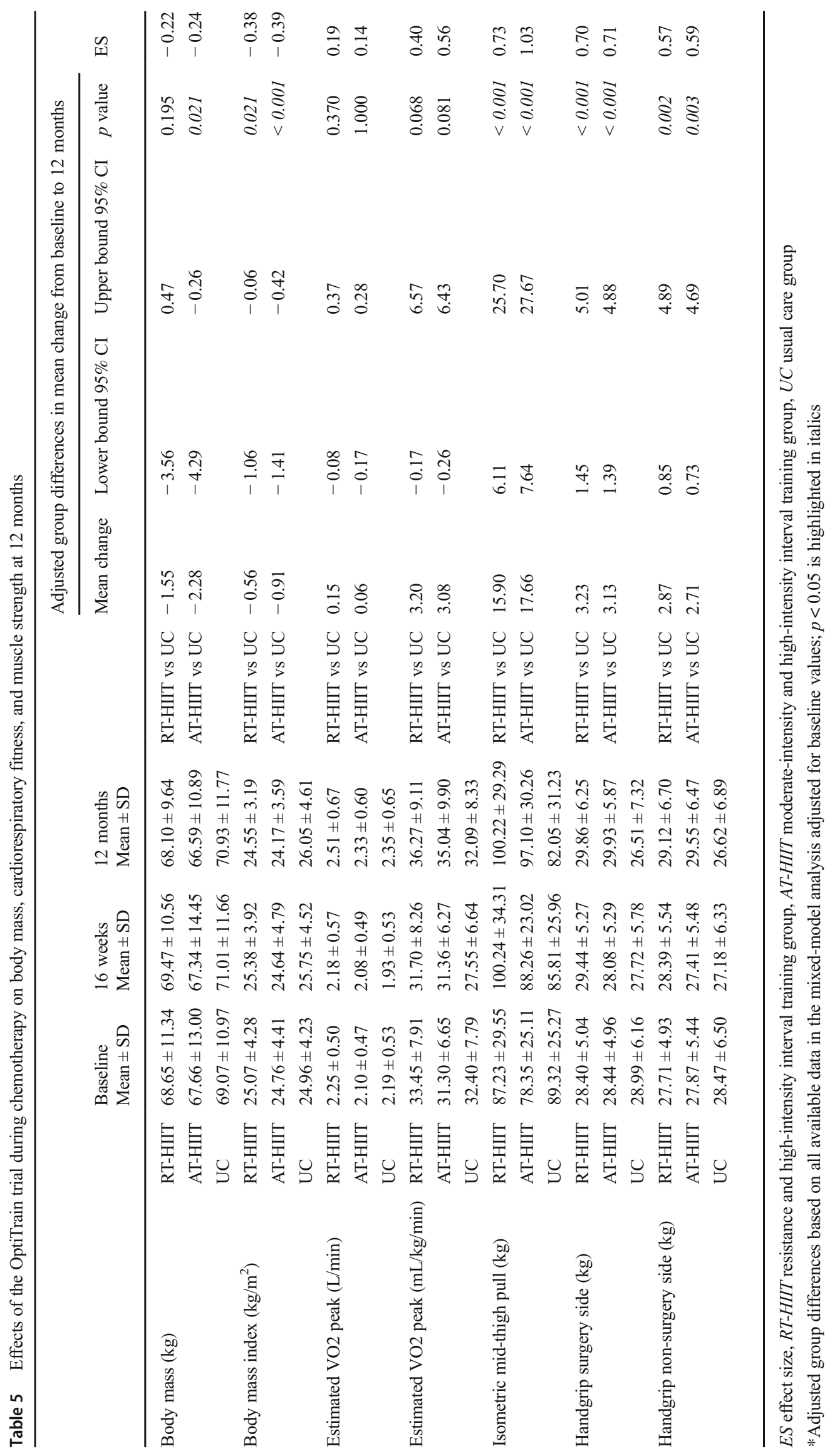


Meeting exercise recommendation guidelines

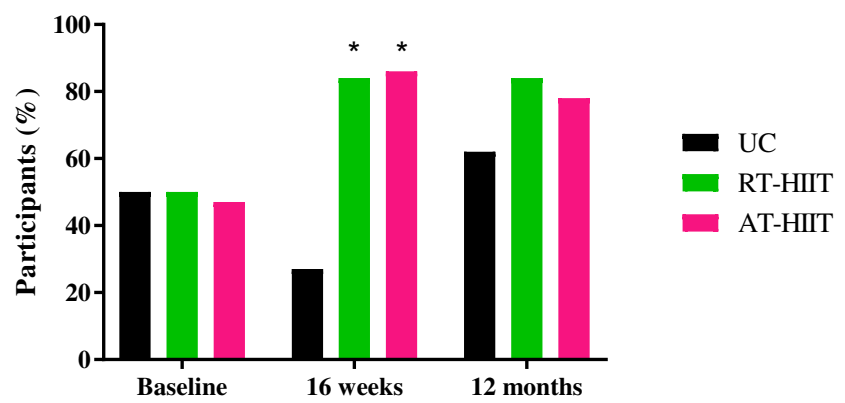

Fig. 2 Percentage of each group reporting meeting exercise recommendation guidelines based on the single item questionnaire at baseline, 16 weeks, and 12 months post-baseline. RT-HIIT, resistance and high-intensity interval training group; AT-HIIT, moderate-intensity aerobic and high-intensity interval training group; UC, usual care group. $* p<0.05$ vs UC

as a factor underlying CRF. Our findings are in line with a cross-sectional study that demonstrated poor lower-extremity muscle strength as one of the predictors of fatigue in long-term breast cancer survivors [34].

The effects of exercise on sick leave/return to work has received increased attention in patients with cancer. In line with our findings, an exercise trial conducted after completion of chemotherapy demonstrated that those who had performed high-intensity exercise had an increased ability to work [35]. Physical and cognitive fatigue/impairments have been shown to affect work capacity, and fatigue 6 months after chemotherapy predicted a longer sick leave [36]. Both exercise groups counteracted fatigue at 12 months, and it may be speculated that the superior effects found for AT-HIIT on sick leave/return to work may be due to the ability to counteract cognitive fatigue compared to the UC group. In addition to experiencing increased cognitive fatigue, although no significant differences were found between groups, the UC group showed lower levels for psychological symptoms and cognitive functioning on the MSAS and EORTC-QLQ-C30 subscales, while other symptoms and quality of life functional-related aspects had returned to baseline levels. This is in line with a recent longitudinal exercise trial for patients with breast cancer showing that cognitive functioning was still impaired while quality of life-related functions improved over time since chemotherapy [37].

The OptiTrain trial is the first to report on exercise effects on symptoms using a symptom-specific scale. The exercise interventions were effective in relieving, and even showing improvements, for multiple dimensions of symptoms at the follow-up. A previous study showed that more than one in four cancer survivors had high symptom burden 1 year post diagnosis [38], emphasizing the important role of exercise interventions during chemotherapy in managing persistent symptoms.
A

Total CRF at baseline

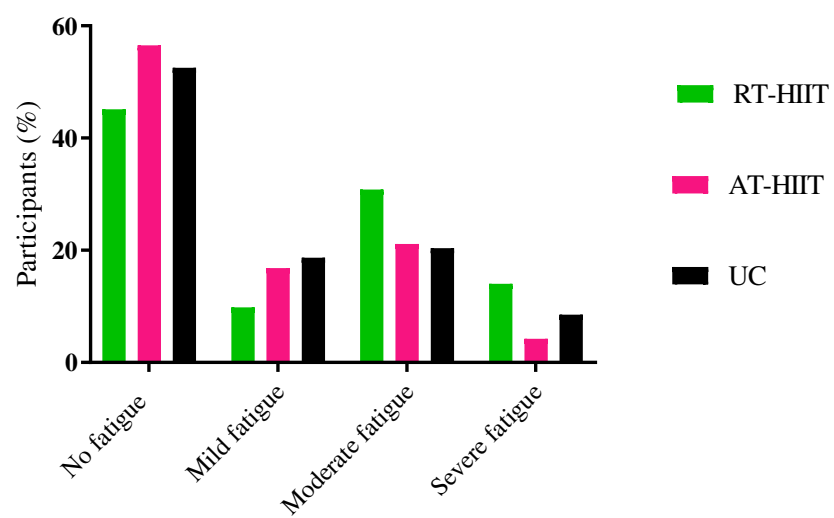

B

Total CRF at 16 weeks

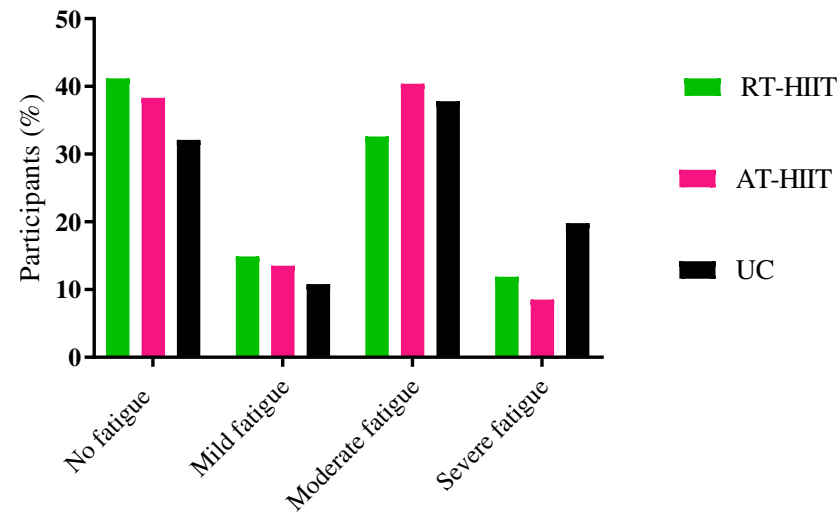

C Total CRF at 12 months

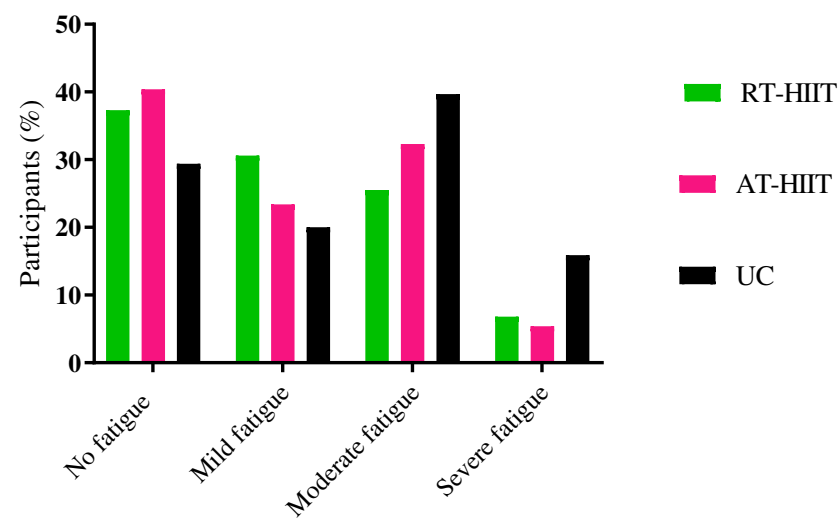

Fig. 3 Total cancer-related fatigue levels divided into categories: no fatigue, mild fatigue (score $>0$ ), moderate fatigue (score $>4$ ), or severe fatigue (score > 7) at a baseline, b 16 weeks, and c 12 months postbaseline. RT-HIIT, resistance and high-intensity interval training group; AT-HIIT, moderate-intensity aerobic and high-intensity interval training group; UC, usual care group; CRF, cancer-related fatigue

Both exercise groups displayed significant strength gains with moderate to large effect sizes. The RT-HIIT group was able to maintain the muscle strength gained during chemotherapy into survivorship, while the AT-HIIT group gained muscle 
strength after chemotherapy to similar levels as the RT-HIIT group at the follow-up. It may be speculated that since the ATHIIT group performed sessions at the same time as RT-HIIT, the AT-HIIT group may have been inspired and incorporated resistance exercise training in their activities after completion of the intervention. A study that assessed PA attendance of breast cancer survivors up to 12 months, after a combined resistance and aerobic exercise intervention, showed that strength exercise was reported to be the most common form of exercise [39]. Nevertheless, few previous trials conducted during chemotherapy have found differences between exercise and control groups at the follow-up [13-15]. A persistent decline in muscle strength is associated with a decline in overall health and quality of life among breast cancer survivors [40] and is a strong independent predictor of all-cause mortality in healthy elderly [41]. Therefore, the implementation of highload resistance exercise during chemotherapy is of major importance as well as motivational support to maintain/implement resistance training after completion of chemotherapy.

Previous findings have shown an average weight gain of $2 \mathrm{~kg}$ in women with breast cancer 1 year after chemotherapy and difficulties to return to pre-diagnosis body weight [42]. Emerging evidence indicate that exercise has a negligible impact on weight loss in healthy individuals [43]. Whether exercise interventions can limit the increase in body mass in patients with breast cancer is largely unknown. Here, the women who had performed exercise during chemotherapy displayed a reduced body mass at the follow-up, with only the AT-HIIT group reaching the significance level compared to the UC group that instead gained weight. Previous trials were unable to show any effects of combined resistance and endurance training on follow-up body weight $[14,17]$ or body composition [15], possibly due to a sustained muscle mass as a result of resistance training.

Given that knowledge and skills of conducting exercise has been shown to be a predictor of exercise adherence after a cancer treatment [44], we speculate that participation in the OptiTrain trial as well as PA prescriptions facilitated the engagement in exercise training after completion of chemotherapy. Barriers to engage in physical exercise, such as lack of knowledge regarding exercise [45], as well as increased fatigue [4] may have been more difficult to overcome in the control group.

Strengths of this study include the limited loss to followup, a high response rate, and in-clinic measurements of objective muscle strength, cardiorespiratory fitness, and body mass. Moreover, treating the UC group as a control group throughout the duration of the follow-up period facilitates the interpretation of the exercise-trial effects. Limitations include a more active sample that completed physiological in-clinic assessments, which introduces a potential selection bias. However, since this also applied to the UC group, it should not influence the between groups comparisons. Other limitations include not measuring PA objectively at all time points, and not having more detailed information regarding type and intensity of exercise performed, which limits our conclusions on whether any change in PA behavior took place. However, objective PA is currently being measured at the 2 -year assessment and will provide valuable insights into activity levels across all groups.

\section{Conclusion}

Both RT-HIIT and AT-HIIT displayed beneficial effects on cancer-related fatigue, symptoms, and muscle strength, 12 months following the commencement of chemotherapy. Additionally, favorable changes in body mass and return to work were observed for the participants that had been part of the AT-HIIT group during chemotherapy. Importantly, we found moderate to large effect sizes for several outcomes, including fatigue. These findings show that participating in a supervised exercise program for women with breast cancer, with support to maintain physical exercise, can induce longterm benefits. These benefits are not limited to the individual, but also leads to societal benefits with reduced costs associated with prolonged sick leave. Strategies are needed to support patients to exercise both independently and with supervision, throughout the cancer survivorship continuum. It is important to create an awareness of the value of exercise and to provide both patients and health professionals with information on available exercise programs, resources, and qualified exercise specialists. Cost-effectiveness studies on supervised exercise programs for patients with breast cancer are warranted. Future research should also focus on evaluating the safety and effectiveness of innovative exercise approaches such as artificial intelligence exercise solutions that are easy to administer and carry out without supervision.

Acknowledgements The authors would like to thank the participants in the OptiTrain trial, Malin Backman (Karolinska Institutet) for collecting questionnaire data, Jurek Waldfogel (Friskis \& Svettis gym center) for his involvement in organizing motivational seminars, and Karolinska University Hospital for use of their facilities.

Funding This study was supported by the Swedish foundations; Cancerfonden, CancerRehabFonden, The Swedish Society for Medical Research, as well as Radiumhemmets Research Funds.

\section{Compliance with ethical standards}

Conflict of interest The authors declare that they have no conflict of interest.

Ethical approval All procedures performed in studies involving human participants were in accordance with the ethical standards of the institutional and/or national research committee and with the 1964 Helsinki declaration and its later amendments or comparable ethical standards. Informed consent was obtained from all individual participants included in the study. 
Informed consent Informed consent was obtained from all individual participants included in the study.

Open Access This article is distributed under the terms of the Creative Commons Attribution 4.0 International License (http:// creativecommons.org/licenses/by/4.0/), which permits unrestricted use, distribution, and reproduction in any medium, provided you give appropriate credit to the original author(s) and the source, provide a link to the Creative Commons license, and indicate if changes were made.

\section{References}

1. Rossi L, Stevens D, Pierga JY, Lerebours F, Reyal F, Robain M, et al. Impact of adjuvant chemotherapy on breast cancer survival: a real-world population. PLoS One. 2015;10(7):e0132853. https:// doi.org/10.1371/journal.pone.0132853.

2. Tao JJ, Visvanathan K, Wolff AC. Long term side effects of adjuvant chemotherapy in patients with early breast cancer. Breast (Edinburgh, Scotland). 2015;24(0 2):S149-S53. https://doi.org/10. 1016/j.breast.2015.07.035.

3. Courneya KS, Friedenreich CM. Physical activity and cancer control. Semin Oncol Nurs. 2007;23(4):242-52. https://doi.org/10. 1016/j.soncn.2007.08.002.

4. Hofman M, Ryan JL, Figueroa-Moseley CD, Jean-Pierre P, Morrow GR. Cancer-related fatigue: the scale of the problem. Oncologist. 2007;12(Suppl 1):4-10. https://doi.org/10.1634/ theoncologist.12-S1-4.

5. Curt GA, Breitbart W, Cella D, Groopman JE, Horning SJ, Itri LM, et al. Impact of cancer-related fatigue on the lives of patients: new findings from the fatigue coalition. Oncologist. 2000;5(5):353-60.

6. Jones LW, Courneya KS, Mackey JR, Muss HB, Pituskin EN, Scott $\mathrm{JM}$, et al. Cardiopulmonary function and age-related decline across the breast cancer survivorship continuum. J Clin Oncol. 2012;30(20):2530-7. https://doi.org/10.1200/jco.2011.39.9014.

7. Peel AB, Thomas SM, Dittus K, Jones LW, Lakoski SG. Cardiorespiratory fitness in breast cancer patients: a call for normative values. J Am Heart Assoc. 2014;3(1):e000432. https://doi.org/ 10.1161/jaha.113.000432.

8. Klassen O, Schmidt ME, Ulrich CM, Schneeweiss A, Potthoff K, Steindorf K, et al. Muscle strength in breast cancer patients receiving different treatment regimes. J Cachexia Sarcopenia Muscle. 2017;8(2):305-16. https://doi.org/10.1002/jcsm.12165.

9. Mijwel S, Backman M, Bolam KA, Jervaeus A, Sundberg CJ, Margolin S, et al. Adding high-intensity interval training to conventional training modalities: optimizing health-related outcomes during chemotherapy for breast cancer: the OptiTrain randomized controlled trial. Breast Cancer Res Treat. 2018;168(1):79-93. https:// doi.org/10.1007/s10549-017-4571-3.

10. Mijwel S, Backman M, Bolam KA, Olofsson E, Norrbom J, Bergh $\mathrm{J}$, et al. Highly favorable physiological responses to concurrent resistance and high-intensity interval training during chemotherapy: the OptiTrain breast cancer trial. Breast Cancer Res Treat. 2018;169(1):93-103. https://doi.org/10.1007/s10549-018-4663-8.

11. Furmaniak AC, Menig M, Markes MH. Exercise for women receiving adjuvant therapy for breast cancer. Cochrane Database Syst Rev. 2016;9:Cd005001. https://doi.org/10.1002/14651858.CD005001. pub3.

12. Courneya KS, Segal RJ, Gelmon K, Reid RD, Mackey JR, Friedenreich CM, et al. Six-month follow-up of patient-rated outcomes in a randomized controlled trial of exercise training during breast cancer chemotherapy. Cancer epidemiology, biomarkers \& prevention: a publication of the American Association for Cancer Research, cosponsored by the American Society of Preventive. Oncology. 2007;16(12):2572-8. https://doi.org/10.1158/10559965.EPI-07-0413.

13. van Waart H, Stuiver MM, van Harten WH, Geleijn E, Kieffer JM, Buffart LM, et al. Effect of low-intensity physical activity and moderate- to high-intensity physical exercise during adjuvant chemotherapy on physical fitness, fatigue, and chemotherapy completion rates: results of the PACES randomized clinical trial. J Clin Oncol. 2015;33(17):1918-27. https://doi.org/10.1200/jco.2014.59. 1081.

14. Travier N, Velthuis MJ, Steins Bisschop CN, van den Buijs B, Monninkhof EM, Backx F, et al. Effects of an 18-week exercise programme started early during breast cancer treatment: a randomised controlled trial. BMC Med. 2015;13:121. https://doi. org/10.1186/s12916-015-0362-z.

15. Haines TP, Sinnamon P, Wetzig NG, Lehman M, Walpole E, Pratt T, et al. Multimodal exercise improves quality of life of women being treated for breast cancer, but at what cost? Randomized trial with economic evaluation. Breast Cancer Res Treat. 2010;124(1): 163-75. https://doi.org/10.1007/s10549-010-1126-2.

16. Juvet LK, Thune I, Elvsaas IKO, Fors EA, Lundgren S, Bertheussen $\mathrm{G}$, et al. The effect of exercise on fatigue and physical functioning in breast cancer patients during and after treatment and at 6 months follow-up: a meta-analysis. Breast. 2017;33:166-77. https://doi.org/10.1016/j.breast.2017.04.003.

17. Mutrie N, Campbell A, Barry S, Hefferon K, McConnachie A, Ritchie D, et al. Five-year follow-up of participants in a randomised controlled trial showing benefits from exercise for breast cancer survivors during adjuvant treatment. Are there lasting effects? J Cancer Surviv. 2012;6(4):420-30. https://doi.org/10.1007/s11764012-0233-y.

18. Piper BF, Dibble SL, Dodd MJ, Weiss MC, Slaughter RE, Paul SM. The revised Piper fatigue scale: psychometric evaluation in women with breast cancer. Oncol Nurs Forum. 1998;25(4):677-84.

19. Jakobsson S, Taft C, Ostlund U, Ahlberg K. Performance of the Swedish version of the revised Piper fatigue scale. Eur J Oncol Nurs. 2013;17(6):808-13. https://doi.org/10.1016/j.ejon.2013.07. 004.

20. Aaronson NK, Ahmedzai S, Bergman B, Bullinger M, Cull A, Duez NJ, et al. The European Organization for Research and Treatment of Cancer QLQ-C30: a quality-of-life instrument for use in international clinical trials in oncology. J Natl Cancer Inst. 1993;85(5):365-76.

21. Browall M, Sarenmalm EK, Nasic S, Wengstrom Y, GastonJohansson F. Validity and reliability of the Swedish version of the memorial symptom assessment scale (MSAS): an instrument for the evaluation of symptom prevalence, characteristics, and distress. J Pain Symptom Manag. 2012;46:131-41. https://doi.org/10.1016/ j.jpainsymman.2012.07.023.

22. Garber CE, Blissmer B, Deschenes MR, Franklin BA, Lamonte MJ, Lee I-M, et al. Quantity and quality of exercise for developing and maintaining cardiorespiratory, musculoskeletal, and Neuromotor fitness in apparently healthy adults: guidance for prescribing exercise. Med Sci Sports Exerc. 2011;43(7):1334-59. https://doi.org/10.1249/MSS.0b013e318213fefb.

23. Schmitz KH, Courneya KS, Matthews C, Demark-Wahnefried W, Galvão DA, Pinto BM, et al. American College of Sports Medicine roundtable on exercise guidelines for cancer survivors. Med Sci Sports Exerc. 2010;42(7):1409-26. https://doi.org/10.1249/MSS. 0b013e3181e0c112.

24. Wengström Y, Bolam KA, Mijwel S, Sundberg CJ, Backman M, Browall $\mathrm{M}$, et al. Optitrain: a randomised controlled exercise trial for women with breast cancer undergoing chemotherapy. BMC 
Cancer. 2017;17(1):100. https://doi.org/10.1186/s12885-017-3079$\mathrm{X}$.

25. Professional Associations for Physical Activity. Physical activity in the prevention and treatment of disease. Stockholm: Swedish National Institute of Public Health; 2010.

26. Morris SB. Estimating effect sizes from pretest-posttest-control group designs. Organ Res Methods. 2007;11(2):364-86. https:// doi.org/10.1177/1094428106291059.

27. Cohen J. Statistical power analysis for the behavioral sciences. 2nd ed. London: Routledge; 1988.

28. Schmidt ME, Wiskemann J, Schneeweiss A, Potthoff K, Ulrich CM, Steindorf K. Determinants of physical, affective, and cognitive fatigue during breast cancer therapy and 12 months follow-up. Int J Cancer. 2018;142(6):1148-57. https://doi.org/10.1002/ijc.31138.

29. van Vulpen JK, Peeters PH, Velthuis MJ, van der Wall E, May AM. Effects of physical exercise during adjuvant breast cancer treatment on physical and psychosocial dimensions of cancer-related fatigue: a meta-analysis. Maturitas. 2016;85:104-11. https://doi.org/10. 1016/j.maturitas.2015.12.007.

30. Kampshoff CS, Chinapaw MJ, Brug J, Twisk JW, Schep G, Nijziel MR, et al. Randomized controlled trial of the effects of high intensity and low-to-moderate intensity exercise on physical fitness and fatigue in cancer survivors: results of the resistance and endurance exercise after chemotherapy (REACT) study. BMC Med. 2015;13:275. https://doi.org/10.1186/ s12916-015-0513-2.

31. Kampshoff CS, van Dongen JM, van Mechelen W, Schep G, Vreugdenhil A, Twisk JWR, et al. Long-term effectiveness and cost-effectiveness of high versus low-to-moderate intensity resistance and endurance exercise interventions among cancer survivors. J Cancer Surviv. 2018;12(3):417-29. https://doi.org/10. 1007/s11764-018-0681-0.

32. Drigny J, Gremeaux V, Dupuy O, Gayda M, Bherer L, Juneau M, et al. Effect of interval training on cognitive functioning and cerebral oxygenation in obese patients: a pilot study. J Rehabil Med. 2014;46(10):1050-4. https://doi.org/10.2340/16501977-1905.

33. Coetsee $\mathrm{C}$, Terblanche E. The effect of three different exercise training modalities on cognitive and physical function in a healthy older population. Eur Rev Aging Phys Act. 2017;14(1):13. https:// doi.org/10.1186/s11556-017-0183-5.

34. Winters-Stone KM, Bennett JA, Nail L, Schwartz A. Strength, physical activity, and age predict fatigue in older breast cancer survivors. Oncol Nurs Forum. 2008;35(5):815-21. https://doi.org/ 10.1188/08.onf.815-821.

35. Thijs KM, de Boer AG, Vreugdenhil G, van de Wouw AJ, Houterman S, Schep G. Rehabilitation using high-intensity physical training and long-term return-to-work in cancer survivors. J Occup Rehabil. 2012;22(2):220-9. https://doi.org/10.1007/ s10926-011-9341-1.
36. de Boer AGEM, Verbeek JHAM, Spelten ER, Uitterhoeve ALJ, Ansink AC, de Reijke TM, et al. Work ability and return-to-work in cancer patients. Br J Cancer. 2008;98:1342-7. https://doi.org/10. 1038/sj.bjc.6604302.

37. Schmidt ME, Wiskemann J, Steindorf K. Quality of life, problems, and needs of disease-free breast cancer survivors 5 years after diagnosis. Quality of life research: an international journal of quality of life aspects of treatment, care and rehabilitation. 2018. https://doi. org/10.1007/s11136-018-1866-8

38. Shi Q, Smith TG, Michonski JD, Stein KD, Kaw C, Cleeland CS. Symptom burden in cancer survivors 1 year after diagnosis: a report from the American Cancer Society's studies of cancer survivors. Cancer. 2011;117(12):2779-90. https://doi.org/10.1002/cncr.26146.

39. Schmidt ME, Wiskemann J, Ulrich CM, Schneeweiss A, Steindorf K. Self-reported physical activity behavior of breast cancer survivors during and after adjuvant therapy: 12 months follow-up of two randomized exercise intervention trials. Acta Oncol. 2017;56(4): 618-27. https://doi.org/10.1080/0284186X.2016.1275776.

40. De Backer IC, Schep G, Backx FJ, Vreugdenhil G, Kuipers H. Resistance training in cancer survivors: a systematic review. Int $\mathrm{J}$ Sports Med. 2009;30(10):703-12. https://doi.org/10.1055/s-00291225330.

41. Newman AB, Kupelian V, Visser M, Simonsick EM, Goodpaster $\mathrm{BH}$, Kritchevsky SB, et al. Strength, but not muscle mass, is associated with mortality in the health, aging and body composition study cohort. J Gerontol A Biol Sci Med Sci. 2006;61(1):72-7.

42. Makari-Judson G, Braun B, Jerry DJ, Mertens WC. Weight gain following breast cancer diagnosis: implication and proposed mechanisms. World J Clin Oncol. 2014;5(3):272-82. https://doi.org/10. 5306/wjco.v5.i3.272.

43. Swift DL, Johannsen NM, Lavie CJ, Earnest CP, Church TS. The role of exercise and physical activity in weight loss and maintenance. Prog Cardiovasc Dis. 2014;56(4):441-7. https://doi.org/10. 1016/j.pcad.2013.09.012.

44. Ormel HL, van der Schoot GGF, Sluiter WJ, Jalving M, Gietema JA, Walenkamp AME. Predictors of adherence to exercise interventions during and after cancer treatment: a systematic review. Psycho-oncology. 2017;27:713-24. https://doi.org/10.1002/pon.4612.

45. Browall M, Mijwel S, Rundqvist $\mathrm{H}$, Wengström Y. Physical activity during and after adjuvant treatment for breast Cancer. Integr Cancer Ther. 2016:1534735416683807. https://doi.org/10.1177/ 1534735416683807 .

Publisher's note Springer Nature remains neutral with regard to jurisdictional claims in published maps and institutional affiliations. 